\title{
Endotoxin Increases Sleep and Brain Allopregnanolone Concentrations in Newborn Lambs
}

\author{
SARAID S. BILLIARDS, DAVID W. WALKER, BENEDICT J. CANNY, AND JONATHAN J. HIRST \\ Department of Physiology, Monash University, Clayton, Victoria, Australia
}

\begin{abstract}
Infection has been identified as a risk factor for sudden infant death syndrome (SIDS). Synthesis of allopregnanolone, a neuroactive steroid with potent sedative properties, is increased in response to stress. In this study, we investigated the effect of endotoxin (lipopolysaccharide, LPS) on brain and plasma allopregnanolone concentrations and behavior in newborn lambs. LPS was given intravenously $(0.7 \mu \mathrm{g} / \mathrm{kg})$ at 12 and $15 \mathrm{~d}$ of age $(n=7)$, and resulted in a biphasic febrile response $(p<0.001)$, hypoglycemia, lactic acidemia $(p<0.05)$, a reduction in the incidence of wakefulness, and increased nonrapid eye movement sleep and drowsiness $(p<0.05)$ compared with saline-treated lambs $(n=5)$. Plasma allopregnanolone and cortisol were significantly $(p<0.05)$ increased after LPS treatment. These responses to LPS lasted $6-8 \mathrm{~h}$, and were similar at 12 and $15 \mathrm{~d}$ of age. Each lamb was then given LPS at $20 \mathrm{~d}$ of age and killed $3 \mathrm{~h}$ posttreatment to obtain samples of the brain. Allopregnanolone concentrations were increased $(p<0.05)$ in all brain areas except the cerebellum and diencephalon. We suggest that
\end{abstract}

ABSTRACT

LPS-induced increase of allopregnanolone in the brain may contribute to somnolence in the newborn, and may be responsible for the reduced arousal thought to contribute to the risk of SIDS in human infants. (Pediatr Res 52: 892-899, 2002)
AS, active sleep
Abbreviations
AW, awake
ECoG, electrocorticogram
EMG, electromyogram
EOG, electrooculogram
GABA, $\gamma$-aminobutyric acid
$\mathbf{G A B A}_{\mathbf{A}}$, GABA-A type receptor
IS, indeterminate sleep
LPS, lipopolysaccharide
QS, quiet sleep
SIDS, sudden infant death syndrome

Infection is a stress often faced by neonates and has been proposed as a risk factor in SIDS. The potential mechanism(s) by which infection could increase susceptibility to SIDS remain unclear. It is likely, however, that a SIDS death results from the coincidence of a number of events that together create a period of increased risk (1). We propose that one of the mechanisms involved is the increased production of sedative neuroactive steroids in response to infection, and that these steroids contribute to the lethargy and increased sleep associated with infection in neonates.

The pathology of infection and its effects on sleep-wake states has been extensively studied (2-4). Together with fever and other "acute phase" changes (such as increased cytokine

Received April 8, 2002; accepted July 30, 2002.

Correspondence: Jonathan J. Hirst, Department of Physiology, Monash University, Clayton, Victoria, Australia 3800, e-mail: jon.hirst@med.monash.edu.au

A National Health and Medical Research Council project grant to J.J.H and D.W.W. and a National Health and Medical Research Council Dora Lush Scholarship to S.S.B. supported this work. Pilot funding for initial work was provided by the Sudden Infant Death Syndrome Foundation of South Australia.

DOI: 10.1203/01.PDR.0000036604.14242.94 production and cortisol levels), increased somnolence is one of the cardinal responses to infection in adults. Several components of the body's response to infection, including increased production of cytokines and prostaglandins, have been shown to induce sleep. Muramyl peptides and LPS associated with the cell wall of bacteria have also been shown to be somnifacient $(5,6)$. Although sleepiness may have an adaptive role that promotes rest and conserves energy, the combination of infection and somnolence in the newborn, with its high metabolic rate and low pulmonary oxygen stores, may increase the susceptibility of the infant to episodes of periodic breathing, sleep-associated apnea, and SIDS by ablating essential cardiorespiratory responses to hypoxia and asphyxia.

Neuroactive steroids are a group of steroids that influence CNS function and, in the adult, can be produced in the brain from cholesterol, or in peripheral organs such as the adrenal cortex and gonads. Progesterone, a key precursor for the synthesis of neuroactive steroids, has been shown to have a number of suppressive effects on brain function. These effects are mediated primarily by $5 \alpha$-reduced metabolites, particularly

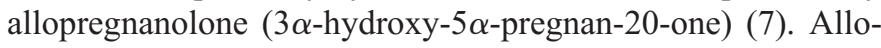


pregnanolone is a positive modulator of the ligand-gated ionotropic $\mathrm{GABA}_{\mathrm{A}}$ receptor, enhancing $\mathrm{GABA}_{\mathrm{A}}$ receptor function by prolonging the channel "open" time and increasing the inward $\mathrm{Cl}^{-}$current, thereby producing sedative, anesthetic, anticonvulsant, and anxiolytic effects $(8,9)$. Studies in the adult rat have shown that the administration of allopregnanolone induces anesthesia $(10,11)$ and alters sleep patterns (7). In addition, it has been suggested that these responses to allopregnanolone may be neuroprotective, particularly in the newborn (12).

We have previously shown that neuroactive steroids have potent suppressive effects on the CNS of fetal sheep (13). We have also found that inhibition of neuroactive steroid production increased arousal-like behavior in the late gestation fetus and reduced the incidence of spontaneous breathing movements (14). These findings suggest that neuroactive steroids increase $\mathrm{GABA}_{\mathrm{A}}$ activity to tonically inhibit the fetal CNS and suppress fetal breathing episodes. The source of steroid precursors in pregnancy is likely to be the placenta. However, it is not clear whether steroidogenesis in the newborn is responsive to stressful stimuli, and whether $5 \alpha$-reduced steroids are produced in sufficient amounts to have physiologic effects on behavior, in particular, on sleep/wake activity.

In this study, we used LPS to mimic the effects of bacterial infection in the newborn lamb. Pregnenolone, progesterone, and allopregnanolone were measured in plasma and the brain. Experiments were repeated three times between 12 and $20 \mathrm{~d}$ of age to allow us to determine whether the responses were attenuated by repeated exposure to LPS, as has been observed in adult animals (15-17). Our results show that large changes in peripheral and central concentrations of allopregnanolone and increased sleep occur on exposure to LPS in the newborn lamb, with no evidence of tolerance.

\section{MATERIALS AND METHODS}

Animals. Twelve lambs of Merino-Border Leicester cross ewes were allowed to deliver spontaneously in lambing pens in the physiology department animal house. The Monash University Standing Committee on Ethics and Animal Experimentation granted prior approval for use of the animals and all procedures performed. All lambs remained with their mother except for the experimental periods described below.

Surgical preparation. Aseptic surgery was performed on all lambs between 5 and $10 \mathrm{~d}$ of age to insert indwelling vascular catheters to obtain blood samples, and to implant electrodes for recording sleep states. Each lamb was deeply anesthetized by inhalation of halothane (Fluothane, ICI Australia Operations, Melbourne, Australia; $1-2 \%$ in $\mathrm{O}_{2}$ ), placed on a heating pad, and given antibiotics (Procaine penicillin, $200 \mathrm{mg}$; dihydrostreptomycin, $250 \mathrm{mg}$ ) by intramuscular injection. A pair of electrodes made from insulated multistranded stainless steel wire (Cooner Wire, Chatsworth, CA, U.S.A.) were implanted through 1-mm holes drilled in the skull over the parietal cortex to record the ECoG. Electrode pairs were also implanted at the inner and outer canthus of the left eye to record EOG, and bilaterally in the dorsal muscles of the neck to record EMG activity. Catheters were placed in a carotid artery and jugular vein for blood sampling and administration of endotoxin, respectively. A saline-filled balloon catheter was inserted into the pleural cavity through a small incision at the fifth intercostal space to measure changes in the rate and depth of breathing from the changes of intrathoracic pressure. All electrodes and catheters were tunnelled under the skin and exteriorized through an incision on the upper back, and then secured beneath a vest of elastic netting. After surgery, lambs recovered in a sling under a heating lamp before being returned to their mother. Experiments did not commence until at least $4 \mathrm{~d}$ after surgery.

Experimental design. All experiments were conducted in a dark, sound-proofed sleep chamber with the lambs resting in a canvas sling. Temperature $\left(22-23^{\circ} \mathrm{C}\right)$ and humidity $(\sim 40 \%)$ were kept constant throughout each study. The sleep chamber allowed for close monitoring of lambs via a video recorder, and this also assisted in the identification of sleep-wake states and other behavioral effects of endotoxin. ECoG, EOG, and nuchal muscle EMG activities together with arterial pressure and intrathoracic pressure were recorded on a polygraph. ECoG, EOG, and EMG activities were recorded using differential AC amplifiers with high-input impedance. Arterial blood pressure was recorded from a pressure transducer located at the level of the heart. Rectal temperature was monitored using a temperature probe inserted at the beginning of each experiment. All signals were displayed on a polygraph (Grass Instruments, Quincy, MA, U.S.A.) using a chart speed of $5 \mathrm{~mm} / \mathrm{min}$. Rate meters triggered by the arterial and intrathoracic pressure waveforms determined heart rate and breathing rate, respectively.

To avoid possible effects of diurnal rhythms, all experiments began at $0800 \mathrm{~h}$. Recordings of sleep-wake states, rectal temperature, and other physiologic variables commenced $1 \mathrm{~h}$ before injection of endotoxin or vehicle. Escherichia coli LPS (0127:B8, Sigma Chemical, St. Louis, MO, U.S.A.) was administered intravenously as a bolus at a dose of $0.7 \mu \mathrm{g} / \mathrm{kg}$ body weight dissolved in $5 \mathrm{~mL}$ of saline ( $n=7$ lambs). Vehicletreated lambs received an i.v. bolus of $5 \mathrm{~mL}$ saline $(n=5$ lambs). Sleep states and physiologic parameters were recorded continuously for $6 \mathrm{~h}$ after LPS or saline treatment.

Arterial blood samples $(4 \mathrm{~mL})$ were collected at $-1,-0.5$, $0,0.5,1,2,3,4,5$ and $6 \mathrm{~h}$, with respect to the saline or LPS injection at time 0 to determine changes of blood gases and $\mathrm{pH}$, and the plasma concentrations of glucose, lactate, cortisol, pregnenolone, progesterone, and allopregnanolone. This 7-h experimental protocol was carried out on two occasions in each lamb, LPS or vehicle treated, at approximately 12 (range, $7-15)$ and 15 (13-18) d of postnatal age. At least 3 d elapsed between these two experiments. Then, at a third and final experiment at approximately 20 (17-21) d of age, each lamb received LPS or vehicle and was killed after $3 \mathrm{~h}$ using i.v. pentobarbitone. The brain was removed immediately $(n=4$ per group), divided into blocks incorporating the cerebellum, midbrain, pons, medulla, diencephalon (thalamus and hypothalamus), hippocampus, frontal, primary, parietal, temporal and occipital cortex. Each block was frozen immediately in liquid nitrogen and stored at $-70^{\circ} \mathrm{C}$ until required for analysis of steroid. 
Data analysis. Identification of sleep-wake states based on the chart records was as previously described $(13,18)$; the videotape recordings further confirmed these results. Briefly, AS was characterized by the presence of rapid eye movements (REM), and absent or reduced nuchal muscle EMG activity during low-amplitude, fast-wave ECoG activity. Lambs in AS also showed increased variability in respiration and blood pressure and occasional twitches of the face and limbs. QS was identified as episodes with reduced or no EOG activity and tonic EMG discharge of the nuchal muscles during highamplitude, slow-wave ECoG. During QS, there was also reduced variation in respiration, blood pressure, and body movements. Stages of sleep that could not be clearly identified as AS or QS, but were nonetheless a "drowsy-like" state, were defined as IS. Wakefulness was characterized by low-amplitude, fast-wave ECoG, with fast and slow eye movements, and tonic and highly variable EMG activity in the neck muscles. A common response to LPS treatment, shivering, was identified by visual observation and the presence of phasic bursts of activity in the nuchal EMG record. A polygraph trace showing the recordings obtained during the pretreatment period and at $3 \mathrm{~h}$ after LPS treatment in one lamb at $21 \mathrm{~d}$ of age is shown in Figure 1. For each experiment, the sleep/wake states of the entire recording were scored at 1-min intervals, and this data combined to obtain an average value for each 30 -min epoch. The proportion (percentage) of the total time spent in a particular state (AS, QS, IS, AW) after saline or LPS treatment was then calculated.

Analytical methods. Arterial blood gases [partial pressure of arterial oxygen $\left(\mathrm{PaO}_{2}\right)$, partial pressure of $\mathrm{CO}_{2}\left(\mathrm{PaCO}_{2}\right), \mathrm{pH}, \mathrm{Hb}$, and oxygen saturation $\left.\left(\mathrm{SaO}_{2}\right)\right]$ were determined using a hemoximeter and an ABL-30 Acid-Base analyzer (Radiometer, Copenhagen, Denmark) with values adjusted for the prevailing rectal temperature of the lamb. Plasma glucose and lactate concentrations were measured using a 2300 STAT analyzer (YSI Inc., Yellow Springs, OH, U.S.A.).

Cortisol concentrations were measured by RIA validated for sheep plasma (19). The minimal detectable level of cortisol was $2.15 \pm 0.22 \mathrm{nmol} / \mathrm{L}(n=10)$. The intra- and interassay coefficients of variance were $4 \%(n=6)$ and $15 \%(n=10)$, respectively.

Neurosteroid steroids were extracted from brain tissue and plasma by a modification of the method of Barbaccia et al. (20), as follows. Frozen brain samples were crushed with mortar and pestle on dry ice, and approximately 100-mg samples were homogenized in $1 \mathrm{~mL}$ (1:10 dilution) ice-cold $50 \%$ methanol containing $1 \%$ acetic acid. The homogenate was then centrifuged at $1800 \times g$ for $15 \mathrm{~min}$ at $4^{\circ} \mathrm{C}$ and the supernatant collected. The pellet was resuspended twice in the above solution and the supernatants were collected, combined, and stored at $-70^{\circ} \mathrm{C}$ until required. Steroids were extracted from plasma and the supernatants from brain homogenates by passing them through a $\mathrm{C}_{18}$ Sep-Pak cartridge (Waters Associates, Milford, MA, U.S.A.), previously equilibrated by washing first with $2.5 \mathrm{~mL} 100 \%$ methanol, then with $2.5 \mathrm{~mL} 50 \%$

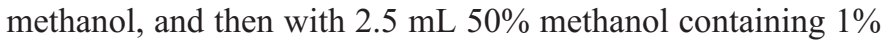
acetic acid. After loading the samples, the cartridge was washed with $50 \%$ methanol containing $1 \%$ acetic acid followed (a)

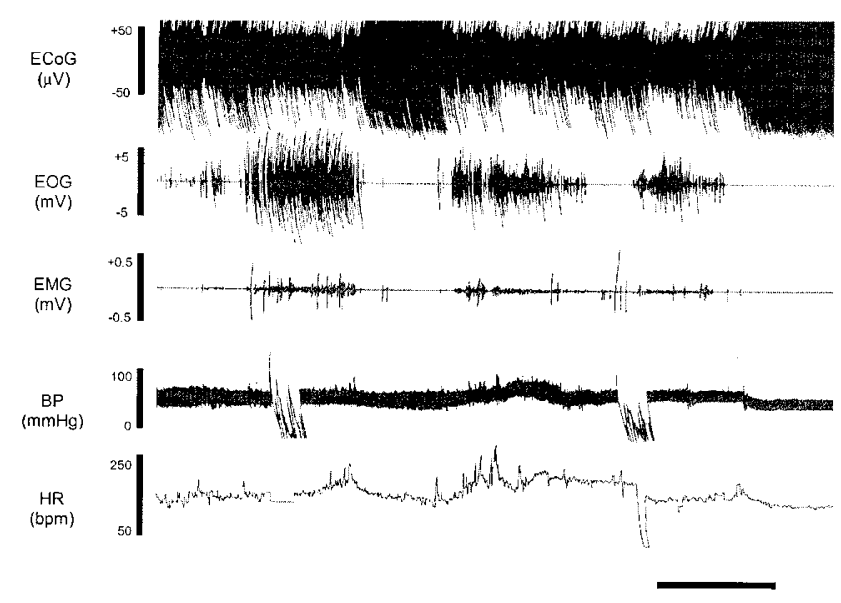

(b)

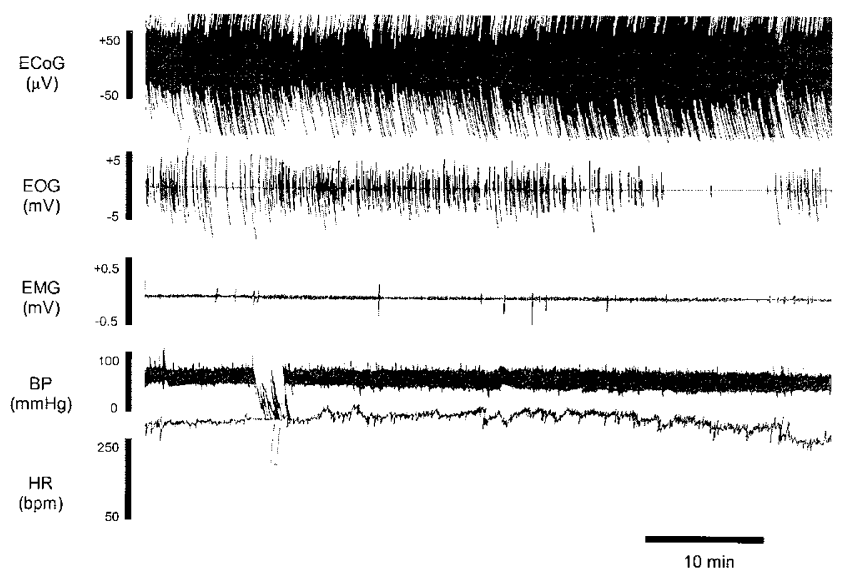

Figure 1. Polygraph recording showing ECoG, EOG, EMG, blood pressure $(B P)$, and heart rate $(H R)$ from a 21-d-old lamb. The record shows $(a) 1 \mathrm{~h}$ of basal activity and $(b) 1 \mathrm{~h}$ of recording $2 \mathrm{~h}$ after LPS treatment $(0.7 \mu \mathrm{g} / \mathrm{kg}$ i.v. $)$. After LPS treatment there is increased incidence of high-amplitude ECoG and EOG activity. Tonic EMG discharge was also observed after LPS treatment, indicating shivering. There was a dramatic increase in heart rate after LPS treatment, however, there was little change in blood pressure between pre- and post-LPS treatment.

by $50 \%$ methanol, and the steroids eluted with $2 \mathrm{~mL} 100 \%$ methanol. The eluent was then brought to dryness under nitrogen and reconstituted in $1 \mathrm{~mL}$ assay buffer $(0.1 \mathrm{M}$ PBS, $\mathrm{pH}$ 7.0). Pregnenolone and progesterone were measured by specific RIA using antibodies provided by ICN Biomedicals (Seven Hills, NSW, Australia) and Dr. J. Malecki (Bairnsdale, Victoria, Australia), respectively, as previously described (21). Allopregnanolone was quantified by a specific RIA using a polyclonal antibody obtained from Dr. R.H. Purdy (San Diego, CA, U.S.A.), which has previously been characterized (22). Additional samples of brain containing tritium-labeled steroid were homogenized to estimate recovery. The recovery of each steroid through the extraction procedure $(70-90 \%)$ was determined for each assay run and the data were corrected for this procedural loss. The limit of detection for pregnenolone was $0.09 \pm 0.02 \mathrm{pmol} /$ tube $(n=6)$, for progesterone was $0.12 \pm$ $0.02 \mathrm{pmol} /$ tube $(n=10)$, and for allopregnanolone was $0.19 \pm$ 
$0.03 \mathrm{pmol} /$ tube $(n=11)$. The intra- and interassay coefficients of variance were as follows: pregnenolone, $5 \%(n=4)$ and $16 \%(n=14)$; progesterone, $8 \%(n=4)$ and $19 \%(n=29)$; allopregnanolone, $5 \%(n=4)$ and $9 \%(n=17)$.

Statistical analysis. Data were analyzed using SPSS software (v 10.0, SPSS, Chicago, IL, U.S.A.). Repeated-measures ANOVA were used to compare treatment, age, and the interaction between treatment and age. Homogeneity of variance was assessed using Levene's test, and the data were transformed into square root or log data before analysis. Where a significant interaction was found between study, treatment, and/or age the least significant difference test was used. Data are presented as mean $\pm \mathrm{SEM} ; p<0.05$ was considered statistically significant.

\section{RESULTS}

LPS treatments at 12 and 15 d of age. Injection of saline had no effect on rectal temperature, whereas LPS resulted in an increase of rectal temperature $(p<0.001)$ that was biphasic (Fig. 2). The peak of the first phase $\left(40.0 \pm 0.2^{\circ} \mathrm{C}\right)$ occurred at approximately $1 \mathrm{~h}$ post-LPS treatment and the peak of the second phase $\left(40.5 \pm 0.3^{\circ} \mathrm{C}\right)$ occurred at approximately $3 \mathrm{~h}$ post-LPS treatment. Thereafter, the rectal temperature decreased until at $6 \mathrm{~h}$ post-LPS treatment it was slightly but significantly greater than in the control period (Fig. 2). The febrile response differed slightly at the two ages: at $12 \mathrm{~d}$ of age the increase of temperature was slightly, but significantly greater at $3-4.5 \mathrm{~h}$ post-LPS compared with the response at $15 \mathrm{~d}$ of age. At $15 \mathrm{~d}$ of age, the increase of temperature at $1 \mathrm{~h}$ post-LPS was greater than at $12 \mathrm{~d}$ of age. However, in all other respects the biphasic change of temperature was remarkably

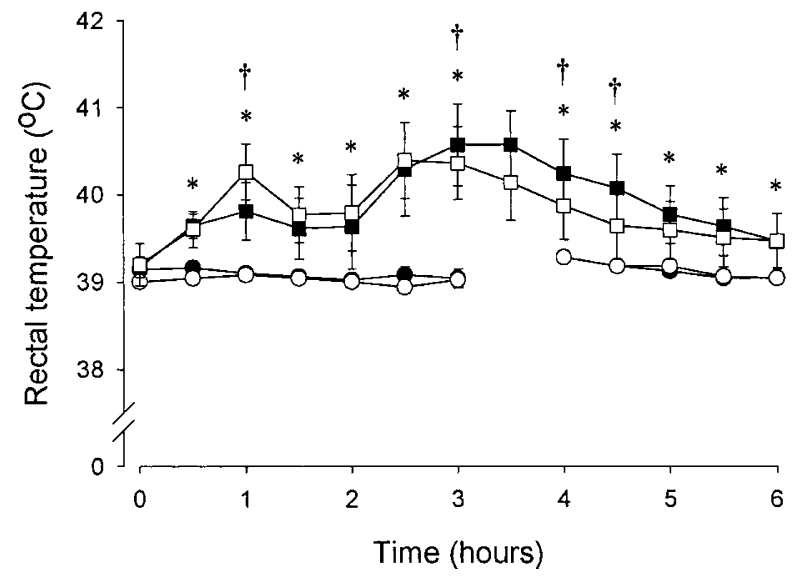

Figure 2. Effect of saline or LPS treatment on rectal temperature. Circles indicate saline treatment at $12 \mathrm{~d}$ (filled circles) and $15 \mathrm{~d}$ (open circles), and squares indicate LPS treatment at $12 \mathrm{~d}$ (filled squares) and $15 \mathrm{~d}$ (open squares) of age. Rectal temperature was measured from $30 \mathrm{~min}$ before treatment at time $(0)$ and continued for $6 \mathrm{~h}$ posttreatment. Pretreatment values did not differ from time (0), and results are presented from this time point. LPS treatment resulted in a biphasic increase of rectal temperature from $30 \mathrm{~min}$ posttreatment at both ages. *Significant differences $(p<0.05)$ between LPS and saline treatments at each time point; $†$ rectal temperatures were different after LPS treatment at 12 and $15 \mathrm{~d}$ of age. Saline treatment had no effect on temperature. The break in the saline data represents the time where lambs had to be returned to the ewe for feeding. Data are shown as mean \pm SEM for 30-min epochs for saline $(n$ $=5)$ and LPS $(n=7)$. similar at the two postnatal ages. LPS treatment also resulted in a marked increase in heart rate and the rate and depth of breathing, which was not different at each of the ages examined (data not shown).

LPS treatment $(n=7)$ resulted in a significant decrease of $\mathrm{SaO}_{2}(96.8 \pm 1.1$ to $92.9 \pm 2.1 \%, p<0.05)$ and $\mathrm{Pao}_{2}(117.3$ \pm 3.48 to $95.4 \pm 6.9 \mathrm{~mm} \mathrm{Hg}, p<0.05)$ at $0.5 \mathrm{~h}$ post-LPS, and of $\mathrm{PaCO}_{2}(36.4 \pm 0.4$ to $31.6 \pm 1.1 \mathrm{~mm} \mathrm{Hg}, p<0.05)$ at $1 \mathrm{~h}$ post-LPS. Saline treatment had no effect on blood gases and $\mathrm{pH}$ $(n=5)$. In the 12-d-old lambs, plasma glucose concentration was increased significantly at 1 and $2 \mathrm{~h}$ after LPS treatment but then decreased markedly $(p<0.05)$ to below control levels by $3 \mathrm{~h}$ posttreatment (Fig. $3 a$ ). In the 15 -d-old lambs, plasma glucose did not increase at $1-2 \mathrm{~h}$ after LPS, but a significant $(p$ $<0.05$ ) reduction was observed by $3 \mathrm{~h}$ after treatment. This reduction in glucose concentration was similar to that observed at $12 \mathrm{~d}$ of age. Plasma lactate concentrations had increased

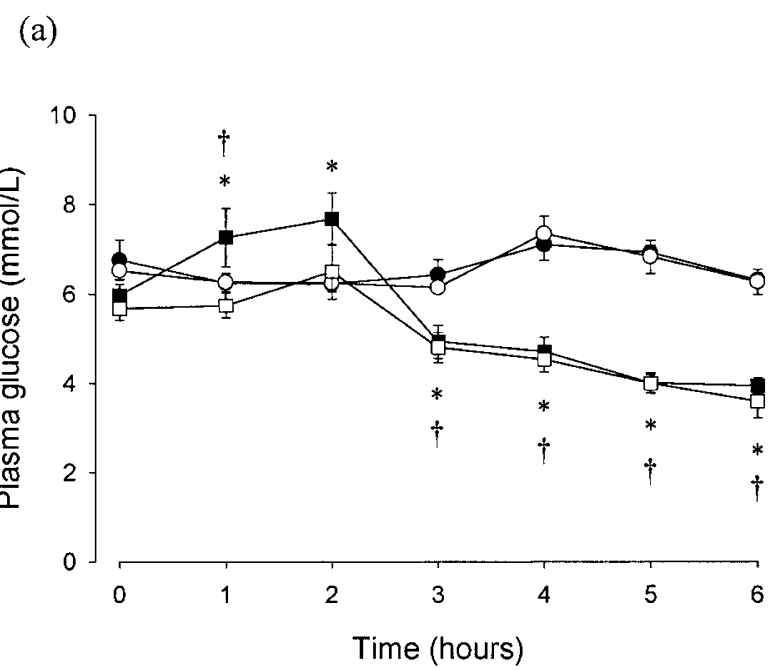

(b)

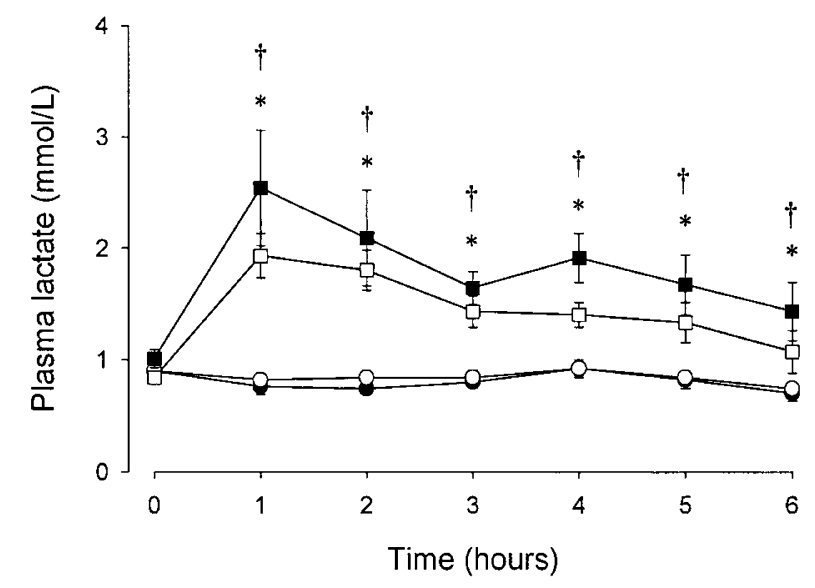

Figure 3. Time course of the effect of saline or LPS treatment at time (0) on plasma glucose $(a)$ and lactate concentrations $(b)$. Circles indicate saline treatment at $12 \mathrm{~d}$ (filled circles) and $15 \mathrm{~d}$ (open circles) and squares indicate LPS treatment at $12 \mathrm{~d}$ (filled squares) and $15 \mathrm{~d}$ (open squares) of age. LPS resulted in a prolonged hypoglycemia and lactic acidemia. Saline had no effect on either parameter. Data shown as mean \pm SEM for saline $(n=5)$ and LPS $(n=7) . * p<0.05$ between saline- and LPS-treated lambs at $12 \mathrm{~d} ; \dagger p<0.05$ between saline- and LPS-treated lambs at $15 \mathrm{~d}$. 
significantly by $1 \mathrm{~h}$ in both experiments at 12 and $15 \mathrm{~d}$ of age $(p<0.001)$ and remained significantly elevated for the duration of the experiment (Fig. $3 b$ ). Plasma glucose and lactate concentrations were not affected by saline treatment.

The duration of the experiments at 12 and $15 \mathrm{~d}$ of age, including the pretreatment period, was $7 \mathrm{~h}$. With saline treatment, the lambs became restless after approximately $4 \mathrm{~h}$ in the sleep chamber (i.e. at $3 \mathrm{~h}$ posttreatment) and had to be returned to the ewe for feeding. This interruption was usually for 15-20 min, hence the recording of temperature and sleep was interrupted at this time. In contrast, LPS-treated lambs remained asleep or were drowsy for the entire 6-h period after endotoxin administration; hence, the record of rectal temperature (Fig. 2) and sleep states for these lambs was continuous. Figure $4 a$ shows the effect of treatment on sleep/wake states for the hour beginning $2 \mathrm{~h}$ after treatment; i.e. immediately before the saline-treated lambs were returned to their mothers. LPS treatment resulted in a significant $(p<0.05)$ decrease in the incidence of wakefulness and increase in the incidence of indeterminate sleep when compared with control animals. When sleep/wake states were calculated as a percentage over the entire 6-h posttreatment period, LPS was also seen to have significantly reduced the incidence of wakefulness and increased the incidence of IS compared with saline-treated lambs (Fig. $4 b ; p<0.05$ ). QS was found to show a tendency toward higher levels after LPS treatment for both analyses, but this did not reach significance (Fig. $4 a: p=0.071$; Fig. $4 b: p=0.095$ ). LPS treatment had no effect on the incidence of AS. There were no differences in the effects of LPS on the incidences of sleep and wakefulness between the experiments at 12 and $15 \mathrm{~d}$ of age for both the hour preceding the return of the salinetreated lambs, or for the entire duration of the experiment.

Plasma allopregnanolone and cortisol concentrations are shown in Figure 5. For experiments at both postnatal ages, plasma allopregnanolone concentrations increased significantly $(p<0.05)$ after LPS treatment, from $1 \mathrm{~h}$ after LPS treatment until the end of the experiment (Fig. 5a). There was no significant change of plasma allopregnanolone after saline treatment. Basal levels of plasma allopregnanolone were also found to decrease with age (see below).

Plasma cortisol concentrations increased approximately 9-fold after LPS treatment $(p<0.05)$. The increase was significant by $1 \mathrm{~h}$ posttreatment $(p<0.05)$ and peak concentrations were observed at approximately $3 \mathrm{~h}$, after which they declined but were still significantly greater than for the saline treatment at $6 \mathrm{~h}(p<0.05)$. In the saline-treated lambs, there was a small but significant change in plasma cortisol levels $(p$ $<0.05$ ), but the concentrations observed at the end of the 6-h period were not different from the pretreatment values (Fig. $5 b)$.

Plasma pregnenolone concentrations were below the level of detection of the assay for the duration of all experiments. Plasma progesterone was undetectable throughout the experiments in the saline-treated lambs, but low plasma concentrations $(\sim 0.03 \mathrm{nmol} / \mathrm{L})$ were detected after LPS treatment (data not shown).

LPS treatment at 20 d of age. In these experiments, the lambs were killed $3 \mathrm{~h}$ after LPS or saline treatment. The effects (a)

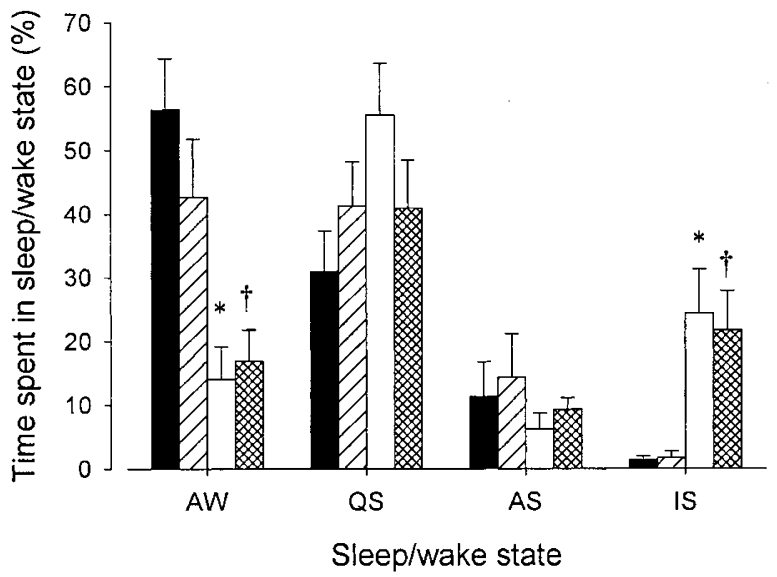

(b)

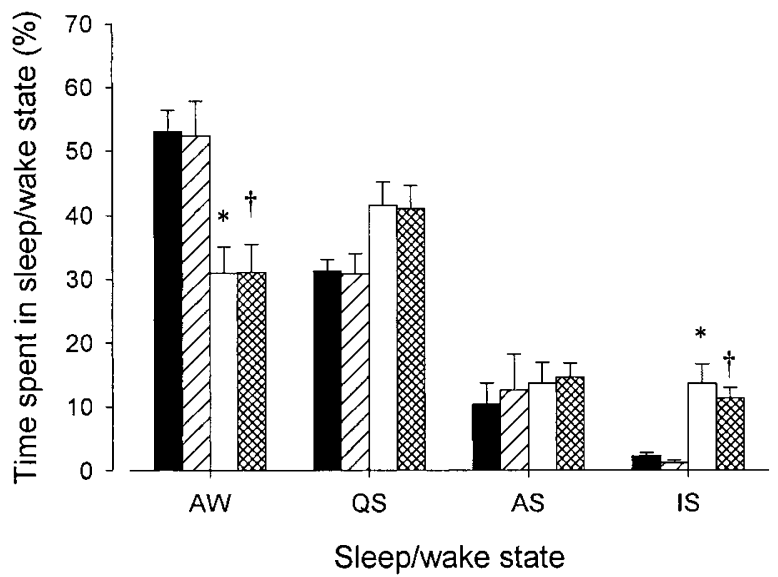

Figure 4. The effect of saline on sleep/wake states in newborn lambs at ( $a$ ) 2-3 h posttreatment and (b) calculated over the duration of the 6-h experiment. Black and hatched bars indicate saline treatment at 12 and $15 \mathrm{~d}$, respectively, and open and checked bars indicate LPS treatment at 12 and $15 \mathrm{~d}$, respectively. Sleep/wake states were quantified from polygraph recordings and visual observation for either $1 \mathrm{~h}(a)$ or $6 \mathrm{~h}(b)$ after saline or LPS treatment. Total time spent in each sleep and wake state was calculated and the values represent the proportion of time (\%) spent in that state. LPS treatment resulted in a decrease in wakefulness and an increase in the incidence indeterminate sleep for both figures. Values represent mean \pm SEM for saline $(n=5)$ and LPS $(n=7) .{ }^{*} p$ $<0.05$ between saline- and LPS-treated lambs at $12 \mathrm{~d} ; \uparrow p<0.05$ between saline- and LPS-treated lambs at $15 \mathrm{~d}$.

of LPS on blood gases, acid-base status, plasma glucose, lactate, cortisol, and sleep/wake states for the time period of 0-3 h were not different to those observed at 12 and $15 \mathrm{~d}$ of age. Resting plasma allopregnanolone concentrations decreased significantly between $12(57.0 \pm 0.7 \mathrm{nmol} / \mathrm{L})$ and $15 \mathrm{~d}$ $(49.0 \pm 0.6 \mathrm{nmol} / \mathrm{L} ; p<0.01)$, and between 15 and $20 \mathrm{~d}(40.1$ $\pm 1.3 \mathrm{nmol} / \mathrm{L} ; p<0.05)$. The relative increase of plasma allopregnanolone after LPS treatment was greater $(92.8 \pm$ $12.1 \% ; p<0.05)$ at $20 \mathrm{~d}$ of age than at either $12(51.9 \pm 8.7 \%)$ or $15 \mathrm{~d}$ of age $(55.6 \pm 7.1 \%)$.

Allopregnanolone concentrations in the different regions of the brain obtained $3 \mathrm{~h}$ after saline or LPS treatment are shown in Figure 6. LPS treatment significantly increased allopreg- 
(a)

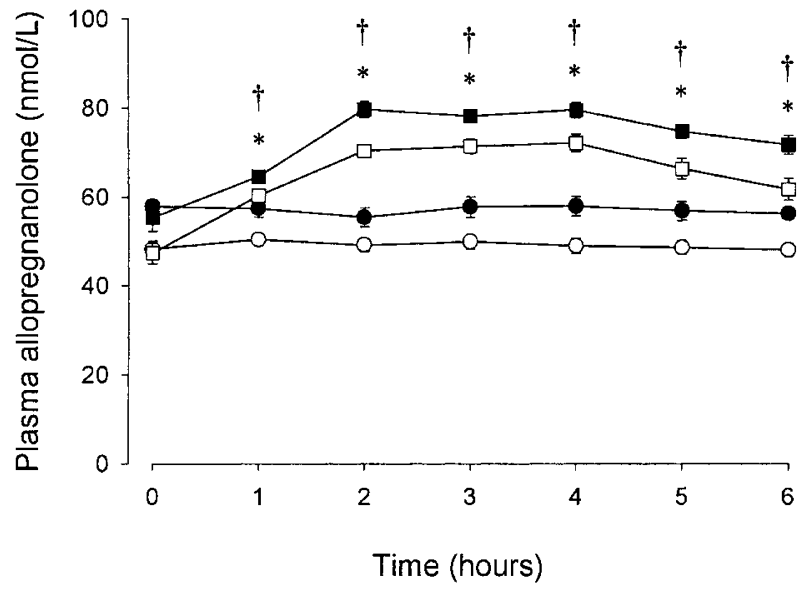

(b)

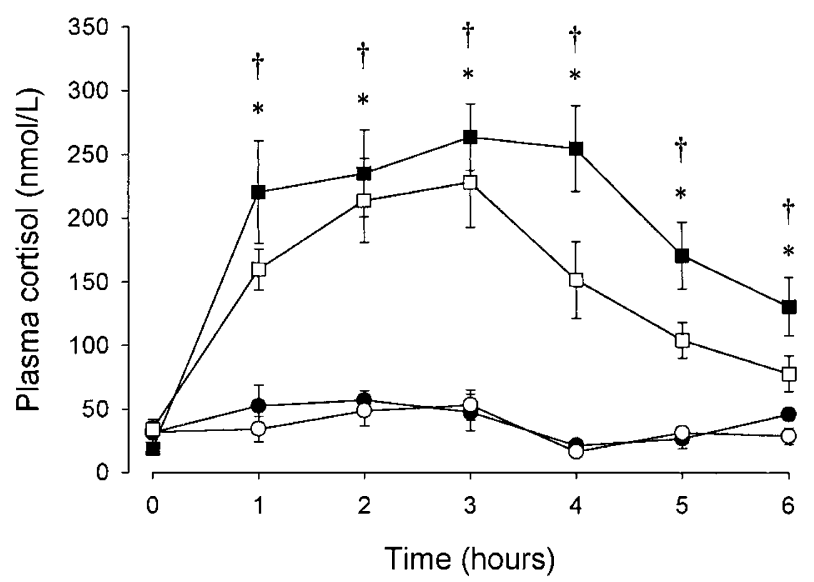

Figure 5. Time course of the effect of saline and LPS on (a) plasma allopregnanolone and $(b)$ cortisol concentrations. Circles indicate saline treatment at $12 \mathrm{~d}$ (filled circles) and $15 \mathrm{~d}$ (open circles), and squares indicate LPS treatment at $12 \mathrm{~d}$ (filled squares) and $15 \mathrm{~d}$ (open squares) of age. Allopregnanolone and cortisol concentrations were significantly elevated after LPS treatment at time (0). Saline treatment had no effect on plasma allopregnanolone concentrations, whereas cortisol levels were slightly increased. Data shown as mean \pm SEM for saline $(n=5)$ and LPS $(n=7)$. ${ }^{*} p<0.05$ between saline- and LPS-treated lambs at $12 \mathrm{~d} ; \dagger p<0.05$ between saline- and LPS-treated lambs at $15 \mathrm{~d}$.

nanolone concentrations in the medulla, pons, midbrain, and hippocampus, compared with the saline-treated controls (Fig. $6 a ; p<0.05$ ). Allopregnanolone concentrations were also markedly increased in all the regions of the cortex in response to LPS treatment (Fig. $6 b ; p<0.05$ ). There was no effect of LPS treatment on allopregnanolone concentrations in the cerebellum and diencephalon. Pregnenolone concentrations were $<0.08 \mathrm{pmol} / \mathrm{g}$ in all brain regions, and there was no significant difference between the LPS- and saline-treated groups. Progesterone concentrations were below the level of detection of the assay for both treatment groups, in all brain regions.

\section{DISCUSSION}

The principal finding of this study was that allopregnanolone concentrations were significantly increased in the brain and (a)

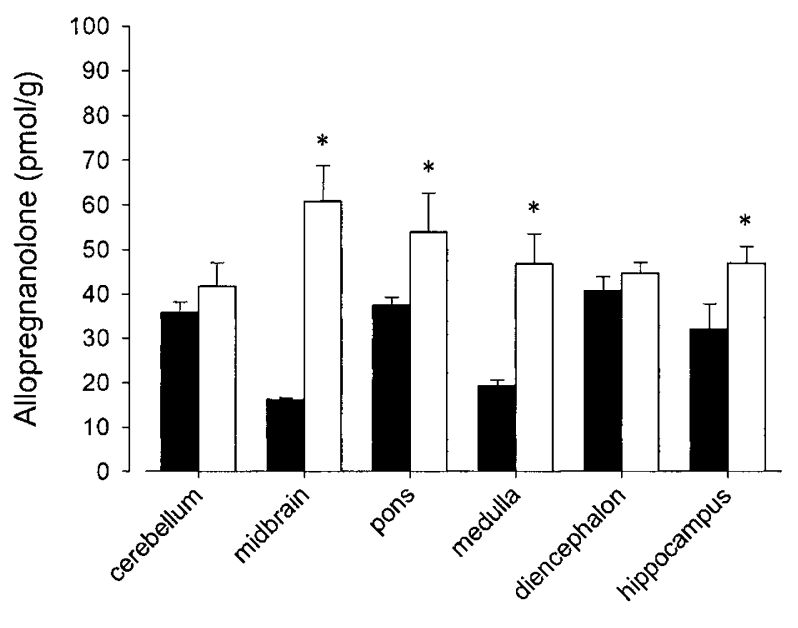

Brain regions

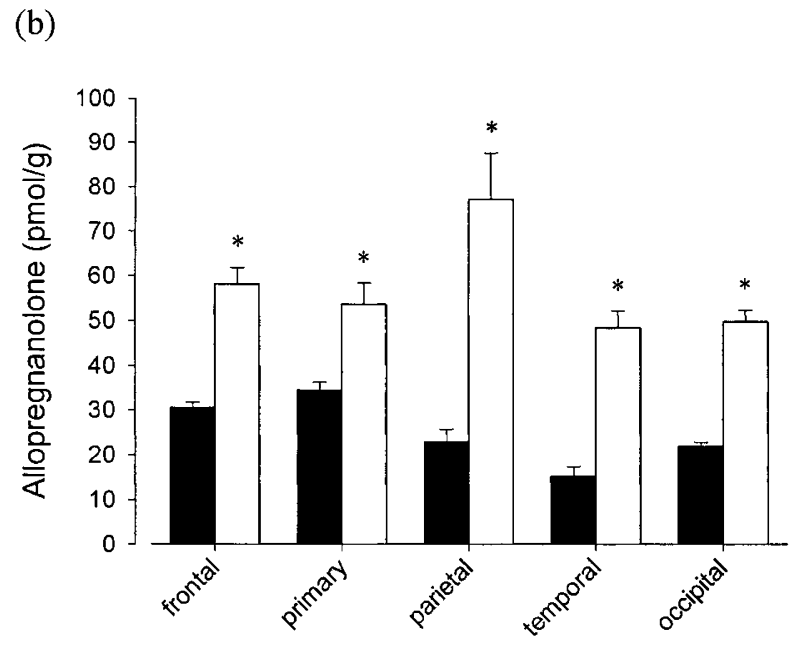

Cerebral cortex

Figure 6. Allopregnanolone concentrations in the major regions of the brain (a) and in specific regions of the cerebral cortex $(b)$ of saline- (filled bars) or LPS-treated (open bars) lambs. LPS treatment resulted in significant increases in allopregnanolone content in most brain regions examined. Values represent mean \pm SEM, $n=4$ for both groups. ${ }^{*} p<0.05$ between saline and LPS treatment at $20 \mathrm{~d}$ of age.

plasma of newborn lambs $3 \mathrm{~h}$ after LPS administration. This coincided with a marked decline in wakefulness and increase in sleep and drowsiness, suggesting a link between stimulated neuroactive steroid production and somnolence in these lambs.

In this study, we used a model of infection that has been used extensively to examine the acute phase response to infection in a number of species. After injection of LPS, we observed the well-established biphasic increase in body temperature (23). This increase has been proposed to result from an initial release of prostaglandins and inflammatory mediators such as tumor necrosis factor- $\alpha$ followed by a more sustained release of cytokines such as IL-1 $\beta$ and IL-6 $(24,25)$. We also observed a moderate biphasic increase in heart rate and respiratory rate, with no consistent change in systemic blood pressure. LPS treatment resulted in an initial oxygen desaturation accompanied by a mild and relatively brief hypocapnia and 
hypoxemia. Plasma glucose levels were significantly lower from $3 \mathrm{~h}$ after LPS treatment compared with the basal period, and lactate concentrations increased in response to LPS administration. No animals died during or after LPS treatment, indicating that the dose of LPS used was adequate to induce a response similar to a relatively mild acute infection.

Acute stressors such as $\mathrm{CO}_{2}$ inhalation and swim stress in adult rats have been shown to lead to increases in neuroactive steroid concentrations in plasma and brain $(26,27)$. The finding that allopregnanolone was elevated in plasma and brain after LPS treatment is consistent with these studies and confirms that neuroactive steroid production is stimulated during stressful events, including infection. The marked rise in plasma cortisol concentrations after LPS treatment is consistent with the activation of the hypothalamo-pituitary-adrenal (HPA) axis (28, 29). Allopregnanolone concentrations have been shown to rise after adrenal stimulation in adult subjects where gonadal production of steroids is prevented, therefore suggesting that the adrenal is the source of plasma allopregnanolone $(30,31)$. We presume that the increase in plasma allopregnanolone concentration observed after LPS treatment results from the stimulation of adrenal secretion, inasmuch as gonadal steroidogenesis would be minimal in these sexually immature lambs.

The increase of allopregnanolone concentrations in the brain was different between regions; from approximately a 4-fold increase in the midbrain and parietal cortex, a 2- to 3-fold increase in other regions of the cerebral cortex, and insignificant changes in the cerebellum and diencephalon. This suggests that there were regionally distinct changes in the in situ production of this steroid in the brain. Some of the increases of brain allopregnanolone were greater, relatively, than the increase of plasma allopregnanolone, also suggesting that the steroid changes observed in the brain are distinct from the peripheral changes of allopregnanolone. The very low concentrations of pregnenolone and progesterone in plasma suggest that delivery of these precursors from the periphery would be unlikely to support the increased cerebral production of allopregnanolone. We have shown that two of the key enzymes involved in the synthesis of allopregnanolone, $\mathrm{P} 450_{\mathrm{scc}}$ and $5 \alpha$-reductase, are strongly expressed in the neonatal brain (32). Although these findings provide support for the role of local production and regulation of allopregnanolone in the newborn brain, it has not yet been established that LPS treatment actually up-regulates either gene expression or enzyme activity.

In the present study, we found that after LPS treatment the lambs were able to remain separated from the ewe for up to $7 \mathrm{~h}$ and that they displayed significantly greater sleep and drowsiness compared with the saline-treated lambs. The salinetreated lambs became restless after $3 \mathrm{~h}$ and were evidently hungry and had to be returned to their mothers. This suggests that one of the effects of LPS is to suppress appetite, as these lambs displayed no sucking reflex or desire to feed from a bottle. Furthermore, the observation that the LPS-treated lambs became hypoglycemic and yet did not display behaviors consistent with hunger is consistent with a possible suppressive effect of LPS on appetite and the desire to feed. At the time when saline-treated lambs were returned to the ewe, they were not hypoglycemic, suggesting that the behavioral response observed was a nonhypoglycemic hunger response or a result of stress at being separated from the ewe. These results support previous findings that LPS administration reduced feed intake in heifers (33) and is consistent with actions of some cytokines (e.g. IL-1 $\beta$ ) in the hypothalamus to induce anorexia (34).

The LPS-treated lambs showed decreased wakefulness and increased drowsiness, whether this was calculated from $1 \mathrm{~h}$ of data immediately before the saline-treated lambs were returned to their mother, or from all of the data obtained posttreatment. Several mechanisms may contribute to the increased sleep that occurs after LPS treatment, such as the induction of sleepinducing prostaglandin $\mathrm{D}_{2}(35)$, as well as a contribution by the increased levels of sedative neuroactive steroids in the brain. Allopregnanolone is a potent sedative steroid with positive modulatory actions with GABA at the $\mathrm{GABA}_{\mathrm{A}}$ receptor, thereby increasing GABAergic inhibition (8). In newborn piglets, dialysis of the $\mathrm{GABA}_{\mathrm{A}}$ agonist, muscimol, into the brainstem disturbs normal sleep patterns and produces a state of drowsiness (36). It is possible that the 2- to 3 -fold increase of allopregnanolone in the brain observed in this study was sufficient to increase sleep, however, we know of no study where the direct infusion of the newborn brain with similar concentrations of allopregnanolone has been associated with increased sleepiness.

To investigate the question of whether the newborn becomes tolerant to infection-induced stimuli, we examined the effect of injecting LPS on three occasions between 10 and $21 \mathrm{~d}$ of age. Previous studies have shown that tolerance develops with repeated doses of endotoxin over a period of days (15-17). However, we found no marked differences between the three LPS treatments in both the temperature and cortisol responses. Differences were observed for the total time spent in sleep/ wake states and the response of plasma allopregnanolone to LPS treatment by the third experiment. However, because the responses to LPS were not diminished at this time, these data suggest that little tolerance to LPS develops with the dose used and with the relatively short-term response generated in these animals.

Basal plasma allopregnanolone concentrations decreased with age in newborn lambs. The cause of this, and its relevance, remains to be elucidated. We have recently found that allopregnanolone concentrations in the brain decrease sharply at birth (37), whereas plasma allopregnanolone concentrations decrease slowly with postnatal age, as shown in the present study. This again suggests a separation of peripheral and CNS production of $5 \alpha$-reduced steroids. It has been proposed that neuroactive steroids such as allopregnanolone may have a neuroprotective function (12), but increases in the newborn brain could be detrimental by creating a higher arousal threshold, and suppression or ablation of responses to hypoxia and apnea, which are problems often faced by the newborn infant.

The contribution of bacterial or viral infection in the etiology of SIDS remains controversial, but is supported by both autopsy and epidemiologic findings (1). The potential link between SIDS and infection is supported by the findings that the prevalence of SIDS is seasonally distributed, with higher death rates occurring in winter months, and several respiratory and 
bacterial infections have been isolated in SIDS victims (38). The pathway by which these infectious challenges contribute to SIDS or apparent life-threatening events may involve several mechanisms. However, it is established that even minor infections lead to marked stimulation of the HPA axis (39) such that a relatively mild infection may cause a marked increase in glucocorticoid concentrations as observed in the present study. Increased glucocorticoid secretion may stimulate neurosteroid synthesis, inasmuch as glucocorticoids are potential precursors for neurosteroid production and may also stimulate the expression of enzymes in the neurosteroidogenic pathway $(30,31)$.

In summary, using small doses of LPS to mimic the effects of infection, we found that concentrations of the sedative steroid allopregnanolone were significantly increased in several brain regions. This coincided with markedly increased plasma cortisol concentrations, an increase in drowsiness, and a decrease in wakefulness. These observations suggest that this steroid may contribute to a reduction in arousal responses that may increase the risk of SIDS after an infectious challenge.

Acknowledgments. The authors thank Mr. Alex Satragno for his assistance with animal care and surgery.

\section{REFERENCES}

1. Filiano JJ, Kinney HC 1994 A perspective on neuropathologic findings in victims of the sudden infant death syndrome: the triple-risk model. Biol Neonate 65:194-197

2. Kadlecova O, Anochina IP, Bauer V, Masek K, Raskova H 1972 Effect of Escherichia coli endotoxin on temperature and sleep cycles of rats. J Infect Dis 126:179181

3. Mullington J, Korth C, Hermann DM, Orth A, Galanos C, Holsboer F, Pollmacher T 2000 Dose-dependent effects of endotoxin on human sleep. Am J Physiol 278:R947R955

4. Toth LA, Krueger JM 1989 Effects of microbial challenge on sleep in rabbits. FASEB J 3:2062-2066

5. Krueger JM, Pappenheimer JR, Karnovsky ML 1982 Sleep-promoting effects of muramyl peptides. Proc Natl Acad Sci U S A 79:6102-6106

6. Krueger JM, Karnovsky ML 1987 Sleep and the immune response. Ann N Y Acad Sci 496:510-516

7. Lancel M, Faulhaber J, Schiffelholz T, Romeo E, Di Michele F, Holsboer F, Rupprecht R 1997 Allopregnanolone affects sleep in a benzodiazepine-like fashion. J Pharmacol Exp Ther 282:1213-1218

8. Barbaccia ML, Roscetti G, Trabucchi M, Mostallino MC, Concas A, Purdy RH, Biggio G 1996 Time-dependent changes in rat brain neuroactive steroid concentrations and $\mathrm{GABA}_{\mathrm{A}}$ receptor function after acute stress. Neuroendocrinology 63:166172

9. Brot MD, Akwa Y, Purdy RH, Koob GF, Britton KT 1997 The anxiolytic-like effects of the neurosteroid allopregnanolone: interactions with $\operatorname{GABA}(\mathrm{A})$ receptors. Eur J Pharmacol 325:1-7

10. Korneyev A, Costa E 1996 Allopregnanolone (THP) mediates anesthetic effects of progesterone in rat brain. Horm Behav 30:37-43

11. Mok WM, Bukusoglu C, Krieger NR 19933 alpha-hydroxy-5 alpha-pregnan-20-one is the only active anesthetic steroid in anesthetized mouse brain. Steroids 58:112-114

12. Kehoe P, Mallinson K, McCormick CM, Frye CA 2000 Central allopregnanolone is increased in rat pups in response to repeated, short episodes of neonatal isolation. Brain Res Dev Brain Res 124:133-136

13. Nicol MB, Hirst JJ, Walker D 1999 Effects of pregnanolone on behavioural parameters and the responses to $\mathrm{GABA}(\mathrm{A})$ receptor antagonists in the late gestation fetal sheep. Neuropharmacology 38:49-63
14. Nicol MB, Hirst JJ, Walker DW 2001 Effect of finasteride on behavioural arousal and somatosensory evoked potentials in fetal sheep. Neurosci Lett 306:13-16

15. Hadid R, Spinedi E, Giovambattista A, Chautard T, Gaillard RC 1996 Decreased hypothalamo-pituitary-adrenal axis response to neuroendocrine challenge under repeated endotoxemia. Neuroimmunomodulation 3:62-68

16. Roth J, McClellan JL, Kluger MJ, Zeisberger E 1994 Attenuation of fever and release of cytokines after repeated injections of lipopolysaccharide in guinea-pigs. J Physiol (Lond) 477:177-185

17. Whyte RI, Warren HS, Greene E, Glennon ML, Robinson DR, Zapol WM 1989 Tolerance to low-dose endotoxin in awake sheep. J Appl Physiol 66:2546-2552

18. Nicol MB, Hirst JJ, Walker D, Thorburn GD 1997 Effect of alteration of maternal plasma progesterone concentrations on fetal behavioural state during late gestation. J Endocrinol 152:379-386

19. Bocking AD, McMillen IC, Harding R, Thorburn GD 1986 Effect of reduced uterine blood flow on fetal and maternal cortisol. J Dev Physiol 8:237-245

20. Barbaccia ML, Roscetti G, Trabucchi M, Ambrosio C, Massotti M 1992 Cyclic AMP-dependent increase of steroidogenesis in brain cortical minces. Eur J Pharmacol 219:485-486

21. Rice GE, Jenkin G, Thorburn GD 1986 Comparison of particle-associated progesterone and oxytocin in the ovine corpus luteum. J Endocrinol 108:109-116

22. Bernardi F, Salvestroni C, Casarosa E, Nappi RE, Lanzone A, Luisi S, Purdy RH, Petraglia F, Genazzani AR 1998 Aging is associated with changes in allopregnanolone concentrations in brain, endocrine glands and serum in male rats. Eur $\mathrm{J}$ Endocrinol 138:316-321

23. Goelst K, Mitchell D, Laburn H 1992 Fever responses in newborn lambs. Pflugers Arch 421:299-301

24. Miller AJ, Luheshi GN, Rothwell NJ, Hopkins SJ 1997 Local cytokine induction by LPS in the rat air pouch and its relationship to the febrile response. Am J Physiol 272:R857-R861

25. Romanovsky AA, Blatteis CM 1995 Biphasic fever: what triggers the second temperature rise? Am J Physiol 269:R280-R286

26. Purdy RH, Morrow AL, Moore PH, Jr., Paul SM 1991 Stress-induced elevations of gamma-aminobutyric acid type A receptor-active steroids in the rat brain. Proc Natl Acad Sci U S A 88:4553-4557

27. Barbaccia ML, Roscetti G, Trabucchi M, Cuccheddu T, Concas A, Biggio G 1994 Neurosteroids in the brain of handling-habituated and naive rats: effect of $\mathrm{CO}_{2}$ inhalation. Eur J Pharmacol 261:317-320

28. Faggioni R, Fantuzzi G, Villa P, Buurman W, van Tits LJ, Ghezzi P 1995 Independent down-regulation of central and peripheral tumor necrosis factor production as a result of lipopolysaccharide tolerance in mice. Infect Immun 63:1473-1477

29. Takeuchi Y, Kikusui T, Kizumi O, Ohnishi H, Mori Y 1997 Pathophysiological changes evoked by lipopolysaccharide administration in goats. J Vet Med Sci 59:125-127

30. Genazzani AR, Petraglia F, Bernardi F, Casarosa E, Salvestroni C, Tonetti A, Nappi RE, Luisi S, Palumbo M, Purdy RH, Luisi M 1998 Circulating levels of allopregnanolone in humans: gender, age, and endocrine influences. J Clin Endocrinol Metab 83:2099-2103

31. Genazzani AR, Bernardi F, Stomati M, Monteleone P, Luisi S, Rubino S, Farzati A, Casarosa E, Luisi M, Petraglia F 2000 Effects of estradiol and raloxifene analog on brain, adrenal and serum allopregnanolone content in fertile and ovariectomized female rats. Neuroendocrinology 72:162-170

32. Petratos S, Hirst JJ, Mendis S, Anikijenko P, Walker DW 2000 Localization of p450scc and 5alpha-reductase type-2 in the cerebellum of fetal and newborn sheep. Brain Res Dev Brain Res 123:81-86

33. Steiger M, Senn M, Altreuther G, Werling D, Sutter F, Kreuzer M, Langhans W 1999 Effect of a prolonged low-dose lipopolysaccharide infusion on feed intake and metabolism in heifers. J Anim Sci 77:2523-2532

34. Sonti G, Ilyin SE, Plata-Salaman CR 1996 Anorexia induced by cytokine interactions at pathophysiological concentrations. Am J Physiol 270:R1394-R1402

35. Hayaishi O 2000 Molecular mechanisms of sleep-wake regulation: a role of prostaglandin D2. Philos Trans R Soc Lond B Biol Sci 355:275-280

36. Darnall RA, Curran AK, Filiano JJ, Li A, Nattie EE 2001 The effects of a GABA(A) agonist in the rostral ventral medulla on sleep and breathing in newborn piglets. Sleep 24:514-527

37. Nguyen P, Billiards SS, Hirst JJ, Walker DW 2002 Ontogeny of allopregnanolone synthesis in the perinatal brain. Proc Aust Neurosci Soc 13:82(abstr)

38. Blackwell CC, Saadi AT, Raza MW, Stewart J, Weir DM 1992 Susceptibility to infection in relation to SIDS. J Clin Pathol 45:20-24

39. Dunn AJ 1993 Role of cytokines in infection-induced stress. Ann N Y Acad Sci 697:189-202 